\title{
Delineating the 15q13.3 microdeletion phenotype: a case series and comprehensive review of the literature
}

\author{
Chelsea Lowther, BSc ${ }^{1,2}$, Gregory Costain, PhD ${ }^{1}$, Dimitri J. Stavropoulos, PhD ${ }^{3,4}$, Rebecca Melvin, BSc', \\ Candice K. Silversides, MD'5,6, Danielle M. Andrade, MD',8, Joyce So, PhD, MD ${ }^{4,9}$, \\ Hanna Faghfoury, MD ${ }^{10}$, Anath C. Lionel, PhD ${ }^{11,12}$, Christian R. Marshall, PhD 11,12, \\ Stephen W. Scherer, PhD ${ }^{2,11,12}$ and Anne S. Bassett, MD ${ }^{1,2,5,13}$
}

\begin{abstract}
Purpose: Recurrent 15q13.3 deletions are enriched in multiple neurodevelopmental conditions including intellectual disability, autism, epilepsy, and schizophrenia. However, the 15q13.3 microdeletion syndrome remains ill-defined.
\end{abstract}

Methods: We systematically compiled all cases of 15q13.3 deletion published before 2014 . We also examined three locally available cohorts to identify new adults with $15 \mathrm{q} 13.3$ deletions.

Results: We identified a total of 246 cases ( 133 children, 113 adults) with deletions overlapping or within the 15 q13.3 (breakpoint (BP)4BP5) region, including seven novel adult cases from local cohorts. No BP4-BP5 deletions were identified in 23,838 adult controls. Where known, $15 \mathrm{q} 13.3$ deletions were typically inherited (85.4\%) and disproportionately of maternal origin $(P<0.0001)$. Overall, 198 cases (121 children, 77 adults; 80.5\%) had at least one neuropsychiatric diagnosis. Accounting for ascertainment, developmental disability/intellectual disability was present in 57.7\%, epilepsy/seizures in $28.0 \%$, speech problems in $15.9 \%$, autism spectrum disorder in $10.9 \%$, schizophrenia in $10.2 \%$, mood disorder in $10.2 \%$, and attention deficit hyperactivity disorder in $6.5 \%$. By contrast, major congenital malformations, including congenital heart disease $(2.4 \%)$, were uncommon. Placenta previa occurred in the pregnancies of four cases.

Conclusion: The 15q13.3 microdeletion syndrome is predominantly characterized by neuropsychiatric expression. There are implications for pre- and postnatal detection, genetic counseling, and anticipatory care.

Genet Med advance online publication 31 July 2014

Key Words: assortative mating; CHRNA7; KLF13; penetrance; TRPM1

\section{INTRODUCTION}

The proximal region of the long (q) arm of chromosome 15 is highly unstable related to six low-copy repeat elements (breakpoint (BP) 1 to BP6). ${ }^{1}$ Distal to the deletions at BP1-BP3 that underlie the Angelman and Prader-Willi syndromes are recurrent 15q13.3 microdeletions (OMIM 612001), typically at BP4-BP5 (Figure 1). ${ }^{2}$ In 2008, Sharp et al. ${ }^{3}$ described the first six cases with 15q13.3 deletions ascertained for intellectual disability (ID), epilepsy, and/or abnormal electroencephalography findings. Since this initial report, deletions at $15 \mathrm{q} 13.3$ have been reported in studies of developmental delay (DD)/ID and/ or multiple congenital anomalies, ${ }^{4}$ epilepsy, ${ }^{5}$ schizophrenia, ${ }^{6}$ autism spectrum disorder (ASD), and other neuropsychiatric disorders. ${ }^{7}$ These findings are consistent with other genomic disorders in which phenotypic variability and incomplete penetrance are common. ${ }^{8,9}$

The use of genome-wide microarrays is now routine in many postnatal settings, ${ }^{10}$ and there is growing interest in the application of this technology to prenatal diagnosis. ${ }^{11}$ This has placed additional pressure on the medical genetics community for phenotypic data across the life span to inform anticipatory care and genetic counseling. The aim of this study was to characterize the individual and collective prevalence of neuropsychiatric conditions in individuals with 15q13.3 microdeletions, as well as to identify new associated features. We conducted an exhaustive search of the literature to identify all previously reported cases and investigated locally available clinical cohorts for adults with 15q13.3 deletions to help delineate the 15q13.3deletion phenotype.

\section{MATERIALS AND METHODS \\ New adult cohorts \\ To further delineate the lifetime expression and penetrance of $15 q 13.3$ deletions, we screened two locally available adult cohorts-one of schizophrenia and chronic schizoaffective dis- order $(n=459)$, and one of tetralogy of Fallot $(n=502)$-for}

\footnotetext{
${ }^{1}$ Clinical Genetics Research Program, Centre for Addiction and Mental Health, Toronto, Ontario, Canada; ${ }^{2}$ Institute of Medical Science, Faculty of Medicine, University of Toronto, Toronto, Ontario, Canada; ${ }^{3}$ Cytogenetics Laboratory, Department of Pediatric Laboratory Medicine, The Hospital for Sick Children, Toronto, Ontario, Canada; ${ }^{4}$ Department of Laboratory Medicine and Pathology, University of Toronto, Toronto, Ontario, Canada; ${ }^{5}$ Division of Cardiology, Department of Medicine, University Health Network, Toronto, Ontario, Canada; ${ }^{6}$ Division of Obstetric Medicine, Medical Disorders of Pregnancy Program, Mount Sinai Hospital, Toronto, Ontario, Canada; ${ }^{7}$ Division of Neurology, Toronto Western Hospital, Toronto, Ontario, Canada; ${ }^{8}$ Krembil Neurosciences Centre, University of Toronto, Toronto, Ontario, Canada; ${ }^{9}$ Department of Clinical Genetics, Lakeridge Health Oshawa, Oshawa, Ontario, Canada; ${ }^{10}$ The Fred A. Litwin and Family Centre in Genetic Medicine, University Health Network and Mount Sinai Hospital, Toronto, Ontario, Canada; ${ }^{11}$ The Centre for Applied Genomics and Program in Genomics and Genome Biology, The Hospital for Sick Children, Toronto, Ontario, Canada; ${ }^{12}$ Department of Molecular Genetics and McLaughlin Centre, University of Toronto, Toronto, Ontario, Canada; ${ }^{13}$ Department of Psychiatry, University of Toronto and University Health Network, Toronto, Ontario, Canada. Correspondence: Anne S. Bassett (anne.bassett@utoronto.ca)
} 


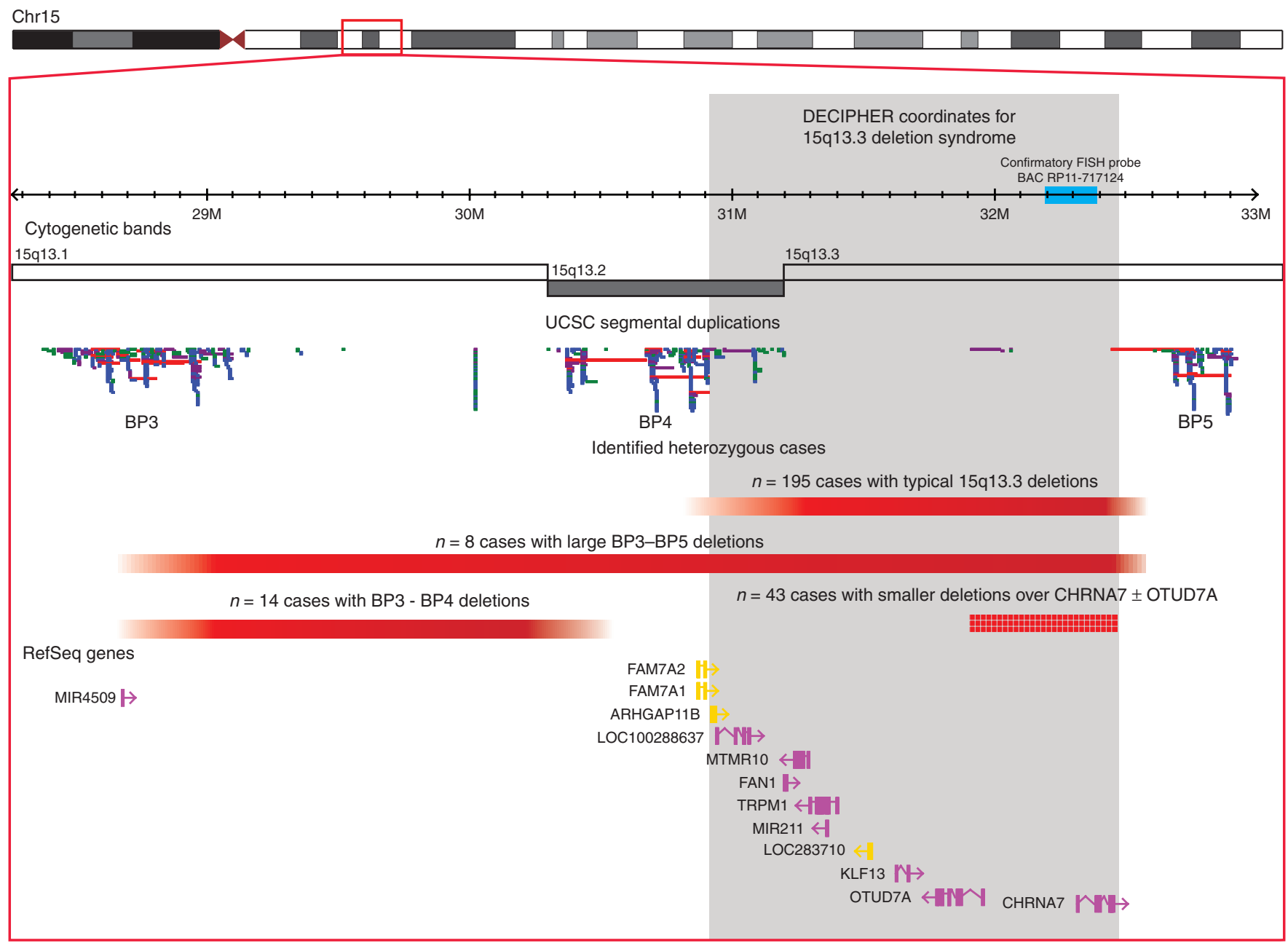

Feb 2009, NCBI B37 (hg19)

Figure 1 Extent of 15q13.1-q13.3 deletion in 260 nonoverlapping cases identified from our centers and the literature. The image was modified from the Database of Genomic Variants (http://dgv.tcag.ca), NCBI Build 37 (hg 19)..$^{40}$ All eight genes (pink) and four pseudogenes (yellow) within the 15q13.3deletion syndrome region (chr15:30,910,306-32,445,407) defined by DECIPHER ${ }^{17}$ (denoted by gray shading), as well as other genes mentioned in this article (e.g., miR-4509) are shown; splice variants are not included. The position of the confirmatory 15q13.3 fluorescence in situ hybridization probe (BAC RP11717124) is outlined in blue above the cytogenetic bands. The recurrent heterozygous 15q13.1-15q13.3 deletions for the cases reviewed, including both children and adults, are represented by red bars. The majority $(n=195)$ of these deletions involved the "typical" 15q13.3 deletion syndrome region (BP4-BP5). Smaller nested deletions within the BP4-BP5 region (represented collectively by a red bar with grid lines) do not tend to have recurrent breakpoints and overlap CHRNA7 only or CHRNA7 and the first exon of OTUDTA.

these deletions. ${ }^{6,12}$ Rare genome-wide copy-number variations (CNVs) were characterized using our established methods. ${ }^{6,12}$ We also searched the Hospital for Sick Children Clinical Cytogenetics Laboratory (Toronto, Canada) database to identify adults with 15q13.3 deletions. The laboratory director (D.J.S.) contacted the referring physicians for potential involvement in our study. Available adult family members of probands with 15q13.3 deletions were also recruited for study. For all local adult cases identified, we obtained genotypic data, performed detailed lifetime chart reviews, and recorded available extended phenotypic data. In addition, for cases 1-4, assessments included a screening questionnaire for lifetime medical and psychiatric history and a modified physical assessment for adults administered by trained clinical research assistants, as previously described. ${ }^{13}$ All data were approved for use by local research ethics boards. To estimate the prevalence of the typical 15q13.3 deletion in the general population, we investigated the 15q13.3 (BP4-BP5) deletion region in control cohorts (total $n$ $=23,838$ adults) with CNV data available from high-resolution genome-wide arrays.

\section{Literature review}

We attempted to identify all individuals with 15q13.1-15q13.3 deletions reported in the literature before 2014. We searched PubMed using the following terms: "15q13 AND (deletion OR loss OR copy number variation)." Second, we examined primary articles and reviews that were not identified by the initial literature search but that pertained to CNV across 10 common conditions: (i) DD/ID and/or multiple congenital anomalies, (ii) epilepsy, (iii) schizophrenia, (iv) ASD, (v) attention deficit 
hyperactivity disorder (ADHD), (vi) major depressive disorder, (vii) bipolar disorder, (viii) Alzheimer disease, (ix) Parkinson disease, and $(\mathrm{x})$ congenital heart disease (CHD). Third, we examined GeneReviews (http://ncbi.nlm.nih.gov/books/ NBK50780/) and the 23 accompanying references.

\section{Inclusion and exclusion criteria for cases identified in the literature review}

We included only those cases with a confirmed interstitial deletion at 15q13.1-q13.3 between BP3 and BP5 (Figure 1). These comprised the "typical" BP4-BP5 $(\sim 1.5-2.0 \mathrm{Mb})$ deletion, the larger $(\sim 3.9 \mathrm{Mb})$ BP3-BP5 deletion, smaller $(<700 \mathrm{~kb})$ deletions nested within the BP4-BP5 region, and proximal BP3-BP4 $(\sim 2.4 \mathrm{Mb})$ deletions that do not overlap the typical BP4-BP5 deletion region (Figure 1). We recorded individuals with smaller or larger heterozygous or homozygous microdeletions in order to potentially define a "critical region" for 15q13.3 microdeletion expression; older reports of large karyotypically visible anomalies were not considered. To determine the phenotypic spectrum of the 15q13.3 deletion, we included only cases for which there was one or more piece of clinical information (e.g., assessment of any physical, cognitive, or developmental feature) other than that used for ascertainment. Cases with only ascertainment data available were excluded. The descriptive terms "normal," "not affected," and "affected" were considered clinical information only if it was clear which clinical feature(s) was being described.

Our initial search revealed 55 primary reports describing cases with 15q13.3 deletions (Supplementary Table S1 online). We systematically reviewed all 55 primary reports and their accompanying supplemental materials and extracted the methodological, genotypic, and phenotypic data in order to determine subject overlap. Individuals from 18 studies were excluded from our analyses because there seemed to be overlap with cases reported elsewhere (see refs. 38-46 in Supplementary Table S1 online) or because the cases reported had only ascertainment data available (see refs. 47-55 in Supplementary Table S1 online). Thus, 37 of 55 studies (67.3\%) contained cases meeting our main inclusion criteria and were used for our analyses.

\section{Clinical definitions}

Any non-ascertainment based feature present in three or more cases was explored as a potential manifestation of the 15q13.3deletion syndrome (Supplementary Table S4 online). DD/ ID was broadly defined to include the spectrum of ID severity (e.g., borderline to severe) and a history of learning difficulties or unspecified cognitive deficits. Macrocephaly and tall stature, and microcephaly and short stature, were defined as physical measurements $\geq 90$ th and $\leq 10$ th percentile, respectively, and were assessed using sex-matched head circumference charts that correct for height ${ }^{14}$ and height-for-age data tables from the Centers for Disease Control and Prevention (http://www.cdc. gov/growthcharts). Prematurity and postmaturity were defined as delivery at $<37$ and $>41$ weeks, respectively. ${ }^{15}$ Large for gestational age ( $\geq 90$ th percentile) and small for gestational age
( $\leq 10$ th percentile) were determined using Canadian population-based references. ${ }^{16}$ Assessment of demographic variables, including basic assumptions used to classify cases as children or adults, and as male or female, was performed using the methods outlined elsewhere. ${ }^{8}$ For each subject, dysmorphic features reported in the originating manuscript or supplemental material were individually coded and analyzed.

\section{RESULTS}

From the local cohorts, we identified seven adults with $15 \mathrm{q} 13.3$ deletions from four families with previously unpublished information on extended phenotype (Table 1). We found one 15 q13.3 deletion (case 1) in our cohort of 459 adults with schizophrenia ${ }^{6}$ and none in our cohort of 502 adults with tetralogy of Fallot. ${ }^{12}$ We tested six relatives of case 1 and identified two sisters and one niece (cases 2-4) with the 15q13.3 deletion (Figure 2). Between February 2008 and December 2013, 11,150 clinical samples were submitted for clinical microarray testing at the Hospital for Sick Children; two adult probands were found to carry a 15q13.3 deletion (cases 5 and 7). A sample from case 6 also was submitted to the Hospital for Sick Children laboratory for parental testing following the birth of an affected child; the 15q13.3 deletion was detected using fluorescence in situ hybridization (BAC RP11-717124).

Together with 253 cases from the literature, we identified 260 individuals (142 children, 118 adults) from 157 families with heterozygous 15q13.1-q13.3 deletions between BP3 and BP5 (Figure 1). Full phenotypic and genotypic details for all children and adults can be found in Table 1 and Supplementary Tables S2 and S3 online. With respect to the extent of deletions, 195 cases $(75.0 \%)$ had the typical BP4-BP5 deletion and $43(16.5 \%)$ had a nested deletion within this 15q13.3 (BP4BP5) region. The nested deletions overlapped CHRNA7 only or CHRNA7 and the first exon of OTUD7A. The larger BP3-BP5 deletion $(n=8 ; 3.1 \%)$ and proximal BP3-BP4 deletion $(n=14$; 5.4\%) were less common (Figure 1). Although underpowered for formal between-group analyses, especially for these latter two recurrent CNVs, we explored the prevalence of individuals with one or more neuropsychiatric feature, including those used for ascertainment, in each deletion subtype. We found no statistically significant differences between the "typical" BP4-BP5 deletions and any of the other three deletion subtypes (data not shown). For the main and nested BP4-BP5 deletions, respectively, the prevalence of these disorders was: $59.0 \%$ and 51.2\% (DD/ID); $29.7 \%$ and $16.3 \%$ (epilepsy); $11.8 \%$ and $4.7 \%$ (schizophrenia); $10.3 \%$ and $14.0 \%$ (ASD); $7.4 \%$ and $11.6 \%$ (ADHD); and collectively for any neuropsychiatric condition $172 / 195$ (89.1\%) and 33/43 (76.7\%).

Of the 14 cases with the $15 \mathrm{q} 13.1-15 \mathrm{q} 13.2$ proximal BP3-BP4 deletion, all 8 probands ( 2 male, 5 female, 1 unspecified sex) were ascertained because of DD/ID; 2 also had seizures and another also had ASD. The six relatives (four transmitting parents, one paternal uncle, and one sibling) were reported as unaffected with respect to DD/ID, although it was unclear how unaffected status was determined. We opted to exclude 


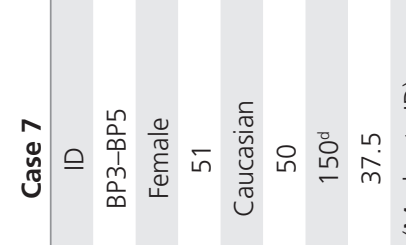

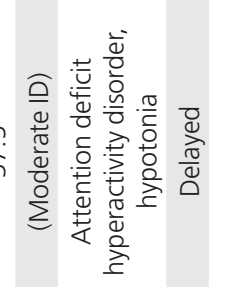

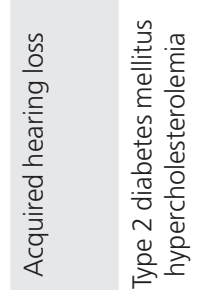

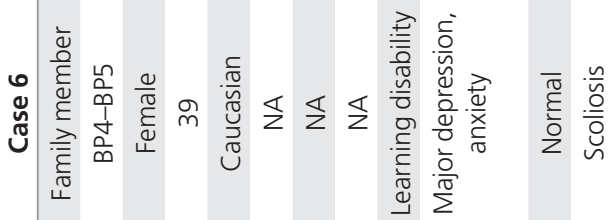

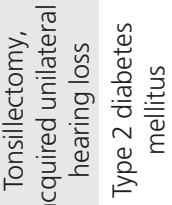

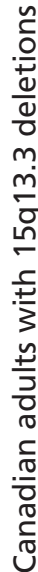
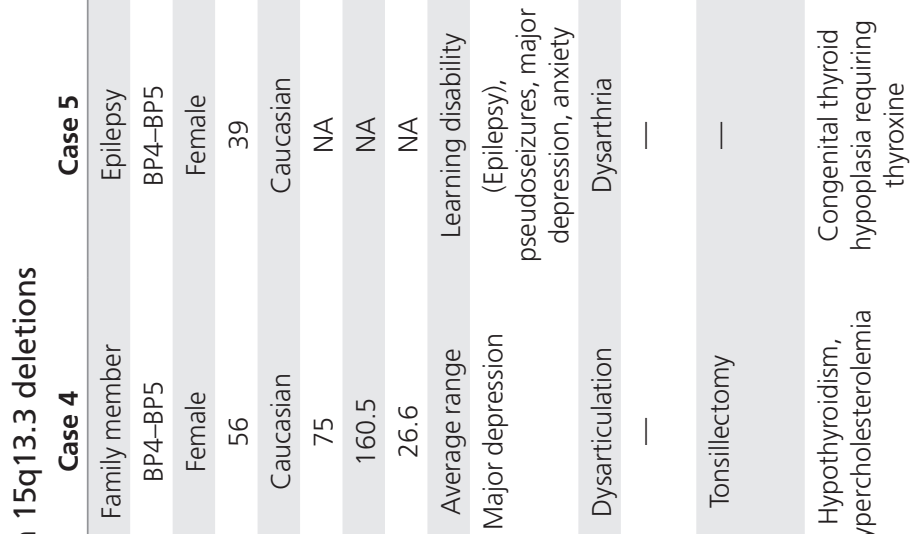

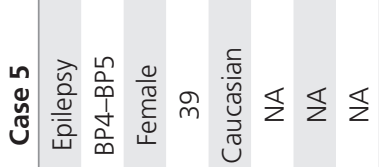

ఖ

4

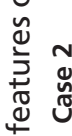

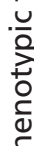

$$
\frac{1}{2}
$$

$\frac{\sqrt{0}}{\frac{0}{2}}$ ำ

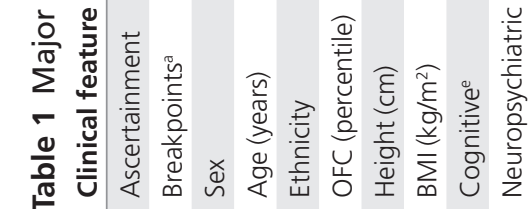
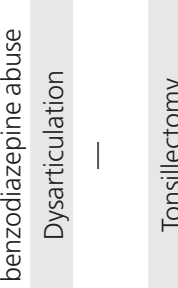

질 ब

हैं

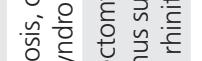
之े $\frac{n}{\sqrt[0]{\frac{C}{0}}}$

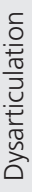

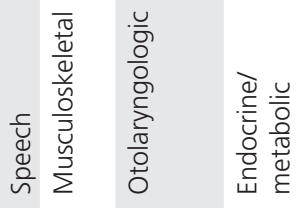

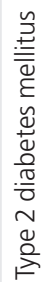

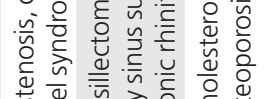

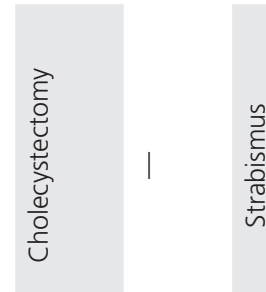

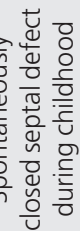

$\stackrel{n}{\frac{n}{n}}$

竞 \begin{tabular}{ll|l}
1 & $\frac{0}{0}$ \\
& $\frac{0}{4}$ \\
& $\frac{0}{4}$
\end{tabular}

$\frac{n}{0}$
$\frac{0}{0}$
$\frac{0}{0}$
$\frac{0}{0}$
$\frac{0}{0}$

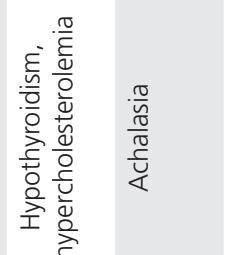

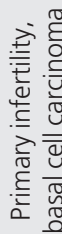
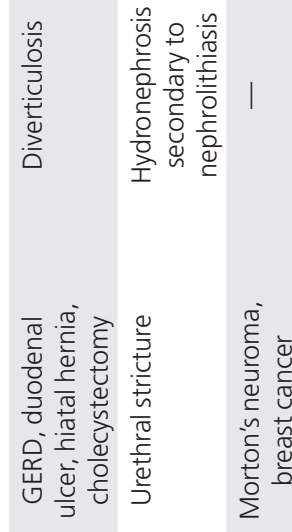

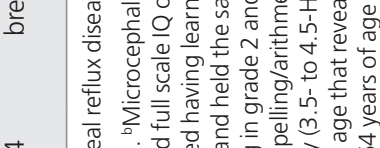

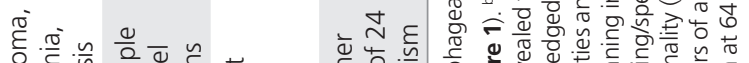

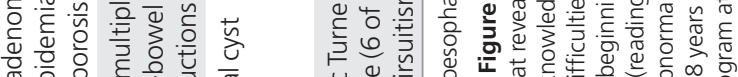

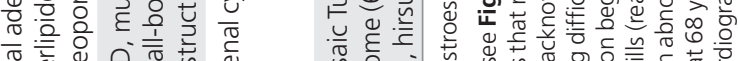

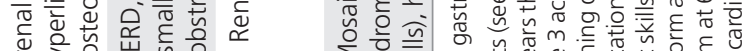
$\sum$ 畜

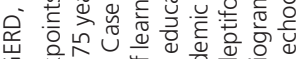

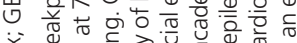

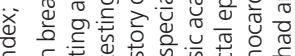

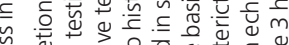

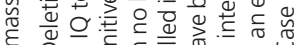

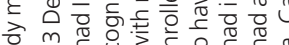

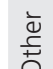



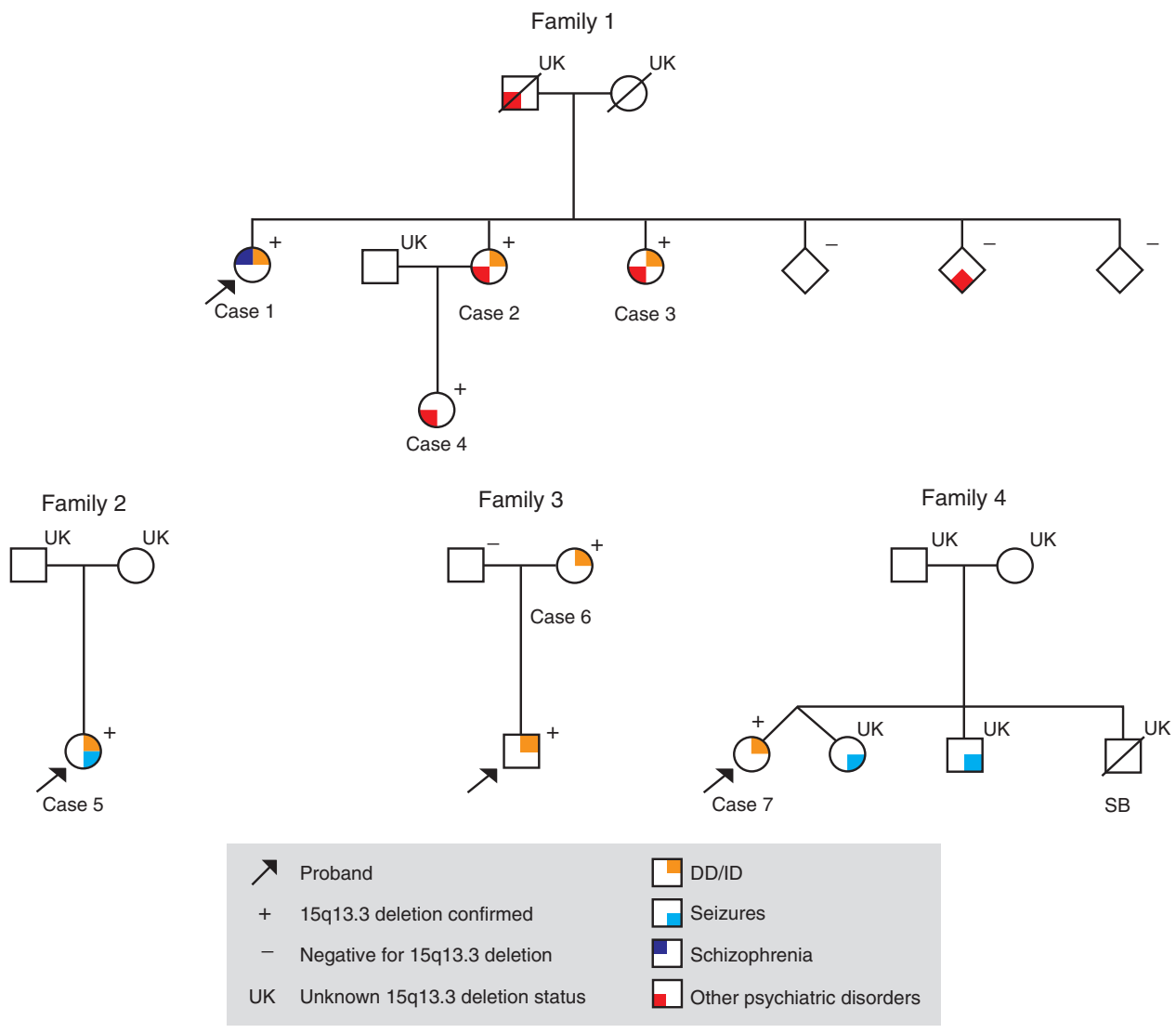

Figure 2 Pedigrees of four new families with the 15q13.3 deletion. Squares and circles represent males and females, respectively. Diamonds indicate either sex, to help preserve anonymity. In Family 1, one sibling was a dizygotic twin of the proband. The strikethrough line denotes deceased status. "UK" represents individuals for whom DNA was not available for testing. Individuals with genetic samples were assayed for deletions at the $15 q 13.3$ locus using microarray or fluorescence in situ hybridization (BAC RP11-717124); results for probands are shown in Table 1. The proband from family 3 was $<18$ years old and thus not included in our study. The case number under individuals is consistent throughout the article. DD, developmental delay; ID, intellectual disability; SB, stillborn.

the BP3-BP4 deletion cases from the main analyses that follow, in order to focus on the 246 cases with deletions that wholly or partly overlapped the 15q13.3-deletion-syndrome region defined by DECIPHER (Figure 1). ${ }^{17}$

\section{5q13.3 Microdeletion expression in children}

There were 133 children (56 boys, 57 girls, 20 unspecified sex) with a heterozygous $15 \mathrm{q} 13.3$ deletion and sufficient phenotype information identified from the literature (Supplementary Table S2 online). The mean reported age was 6.7 (SD 4.8) years (for $n=67$ cases with age data available). Of the 91 of 133 children with data available, the majority of the deletions were inherited $(n=77)$ as compared with de novo $(n=14)$; an excess of the inherited deletions were of maternal origin $(n=55$ of $77 ; 71.4 \%)$. However, there was no apparent sex transmission bias (26 male, 25 female, 4 unknown sex $)\left(\chi^{2}=0.0196 ; \mathrm{df}=1\right.$; $P=0.8886)$ observed in individuals with maternally inherited $15 \mathrm{q} 13.3$ deletions.

The majority of the children $(n=105 ; 79.0 \%)$ were probands who were ascertained because of DD/ID and/or multiple congenital anomalies ( $n=64$ of $105 ; 61.0 \%)$, epilepsy ( $n=31$ of $105 ; 29.5 \%)$, or $\operatorname{ASD}(n=10$ of $105 ; 9.5 \%)$. The remaining 28 children were ascertained as relatives of probands. Including multiple features per subject, 89 children (66.9\%) had DD/ID, 49 (36.8\%) had epilepsy or seizures, and $25(18.8 \%)$ had ASD or pervasive developmental disorder. Only 27 children (30.3\%) had intelligence quotient scores supporting the diagnosis of DD/ID.

After excluding the clinical features used for ascertainment and restricting to conditions present in three or more cases, "behavioral problems" ( $n=46 ; 34.6 \%)$ were the most prevalent features in children with 15q13.3 deletions. These included ADHD ( $n=12 ; 9.0 \%)$, aggression/rage $(n=8)$, hyperactivity $(n=6)$, self-mutilation $(n=4)$, poor concentration $(n=3)$, poor attention $(n=2)$, impulsivity $(n=1)$, and other features $(n=10)$. Including multiple features per subject, 30 (22.6\%) had speech problems, 18 (13.5\%) had hypotonia, $10(7.5 \%)$ had brain abnormalities on magnetic resonance imaging, and $12(9.0 \%)$ had a mood disorder. Just 4 (3.0\%) had CHD. Three (2.3\%) cases had other congenital malformations (Supplementary Table S2 online).

For the 55 (41.4\%) with occipital frontal circumference measurements or percentiles reported, 10 had microcephaly and 15 had macrocephaly. Fifty-three children (39.8\%) had height 
measurements or percentiles reported: 14 had short stature and 13 had tall stature. Of 48 children with weight measurements, 7 were underweight and 15 were overweight or obese. Similar to previous reports, ${ }^{4,18}$ we were unable to identify any specific pattern of dysmorphic features for the 15q13.3 deletion (Supplementary Tables S2 and S3 online).

\section{Children with homozygosity of 15q13.3 microdeletions}

We also identified eight children (six male, two female), including one sibling pair, ${ }^{19}$ with homozygosity of $15 \mathrm{q} 13.3$ deletions born to 14 nonconsanguineous parents (reviewed by MasurelPaulet et al. ${ }^{20}$ ). All eight children had severe to profound DD/ ID and hypotonia. Seven children had seizures, with onset between infancy and 3 years. Six children older than age 4 years were nonverbal. For all seven children with data, visual impairment was reported; three had evidence of retinal dysfunction supported by electroretinography. There were 11 parents with a confirmed heterozygous 15q13.3 deletion and clinical data available who were included in our analyses. Of these, 4 (36.4\%) had a neuropsychiatric condition reported: one mother and one father with mild ID, one mother with mild ID and an abnormal electroencephalography, and one father with an unspecified learning disability and history of febrile seizures during childhood. The remaining seven transmitting parents were described as developmentally and psychiatrically normal, although no formal assessment was provided.

\section{5q13.3 Microdeletion expression in adults}

There were 113 adults (63 women, 42 men, 8 unknown sex) with sufficient phenotypic data identified from the literature (Supplementary Table S3 online) and local cohorts (Table 1). Exact age was available for 25 adults (mean 39.6 [SD 17.1] years). The majority of the $15 \mathrm{q} 13.3$ deletions were of unknown inheritance $(n=92)$. More than half (62 of $113,54.9 \%)$ of the adults were ascertained as transmitting parents; significantly more were mothers $(n=40)$ than fathers $(n=22)\left(\chi^{2}=5.225 ; \mathrm{df}\right.$ $=1 ; P=0.022)$. Of the 62 transmitting parents, $31(50.0 \%)$ were diagnosed with a neuropsychiatric condition; none had schizophrenia. The remaining cases were ascertained as relatives other than parents $(n=12,10.6 \%)$ or as probands diagnosed with a neuropsychiatric condition: schizophrenia $(n=25,22.1 \%)$; $\mathrm{DD} / \mathrm{ID}(n=8,7.1 \%)$; epilepsy $(n=5,4.4 \%)$; or ASD/pervasive developmental disorder $(n=2,1.8 \%)$. Adults not ascertained as transmitting parents showed no significant sex difference (23 women, 20 men; $\chi^{2}=0.209 ; \mathrm{df}=1 ; P=0.647$ ).

Including multiple features per subject, the most common conditions among adults were ID $(n=53,46.9 \%)$, schizophrenia $(n=25,22.1 \%)$, epilepsy and/or seizures $(n=20,17.7 \%)$, mood disorders $(n=13,11.5 \%)$, speech problems $(n=9,8.0 \%)$, aggression/rage $(n=6,5.3 \%)$, and $\operatorname{ADHD}(n=4,3.5 \%)$. Only 10 adults (18.2\%) had intelligence quotient scores supporting the diagnosis of DD/ID. Although none were found in the literature reviewed, endocrine/metabolic conditions were found in all seven local cases (Table 1). Two adults (1.8\%) had CHD; none had other congenital anomalies. For 19 adults (16.8\%) with occipital frontal circumference data, 5 had microcephaly and 3 had macrocephaly. Nineteen adults had height data: eight had short stature and one tall stature. Body mass index could be calculated for only 6 adults (5.3\%); only one was obese and none were underweight. For adults, as for children, we were unable to identify any consistent pattern of dysmorphic features for the $15 \mathrm{q} 13.3$ deletion.

Overall 15q13.3 microdeletion transmission and phenotype Collectively, for children and adults, available data indicated that the majority of the $15 \mathrm{q} 13.3$ deletions were inherited ( $n=94$ of $110 ; 85.4 \%)$; significantly more were of maternal as compared with paternal origin $\left(n=69\right.$ vs. $n=25 ; \chi^{2}=33.0$; $\mathrm{df}=1 ; P<0.0001)$. By not restricting our analysis to ascertainment data, 198 (121 children, 77 adults; $80.5 \%$ ) of the total 246 cases had a neuropsychiatric diagnosis: DD/ID $(n=142$, $57.7 \%)$; epilepsy or seizures $(n=69,28.0 \%)$; speech problems $(n=39,15.9 \%)$; ASD $(n=27 ; 10.9 \%)$; schizophrenia $(n=25$; $10.2 \%)$; mood disorders $(n=25,10.2 \%)$; and $\operatorname{ADHD}(n=16$; $6.5 \%)$. CHD was identified in just 6 cases (2.4\%).

\section{Placenta previa potentially associated with the $15 q 13.3$ microdeletion}

Of the 246 individuals with 15q13.3 deletions, 27 (11.0\%) had adverse perinatal outcomes reported (Supplementary Table S5 online). The majority $(n=23)$ of the scenarios pertained to an offspring carrying the 15q13.3 deletion, and the remaining 4 pertained to pregnancy/obstetrical issues in transmitting mothers with the 15q13.3 deletion. The most common serious perinatal outcome reported was placenta previa $(n=4)$, meeting our criteria as a potential manifestation of the 15q13.3-deletion syndrome.

\section{Prevalence of 15q13.3 region deletions in control populations}

Data on 23,838 adult control cases revealed no BP4-BP5 deletions (Supplementary Table S6 online). The control dataset was not examined for BP3-BP5, BP3-BP4, or nested deletions within the 15q13.3 (BP4-BP5) region. Two additional studies reporting on a total of 4,480 controls also found no $15 \mathrm{q} 13.3$ deletions (Supplementary Table S6 online). By contrast, a recent report of a population-based sample $(n=101,655)$ representing one-third of the Icelandic population identified 25 BP4-BP5 deletions $(0.025 \%), 8$ deletions $(0.0078 \%)$ overlapping CHRNA7 only, and 4 BP3-BP4 deletions $(0.0039 \%))^{21}$ There were seven 15q13.3 (BP4-BP5) duplications found among our 23,838 controls $(0.029 \%)$, consistent with previous reports. ${ }^{3,5}$

\section{DISCUSSION}

We report the results of the single largest review of $15 q 13.3$ deletions to date, involving 246 cases with deletions overlapping the 15 q13.3 BP4-BP5 region, including previously unpublished data for 7 adults. Expanding analyses beyond ascertainment data allowed new insights into the expressivity and penetrance 
of these deletions. The main findings support the predominance of neurodevelopmental/neuropsychiatric conditions, including DD/ID, epilepsy, speech problems, ASD, schizophrenia, mood disorders, and ADHD. ${ }^{4-6,18,22}$ The penetrance for these conditions collectively is high, approaching $80 \%$.

By contrast, penetrance was low for major congenital anomalies, including $\mathrm{CHD}$, supported by the absence of $15 q 13.3$ deletions in genome-wide microarray studies of CHD (Supplementary Table $\mathbf{S 1}$ online), including ours. ${ }^{12}$ The results suggest that fetal anomalies on ultrasonography would be expected to be rare, consistent with the low prevalence of $15 q 13.3$ deletions detected by prenatal chromosomal microarray studies. ${ }^{11,23,24}$ Postnatal chromosomal microarray detection, perhaps secondary to referral for DD/ID, is higher. ${ }^{9}$ By contrast, the most common large rare CNVs, 22q11.2 deletions, have high penetrance for both neuropsychiatric disorders and congenital anomalies, ${ }^{25}$ and higher prenatal detection $(0.29 \%)$ as compared with $15 \mathrm{q} 13.3$ deletions $(0.02-0.09 \%) .{ }^{26}$ In the absence of newborn screening data, however, the true live birth prevalence for all pathogenic CNVs is unknown.

Homozygous or compound heterozygous 15q13.3 deletionsnow numbering 11 (refs. 20 and 27) - are unique among pathogenic CNVs and provide further support for the predominance of neuropsychiatric expressivity. These highlight the issue of assortative mating, perhaps related to neuropsychiatric phenotype. Across the entire sample, however, there were significantly more mothers than fathers who transmitted the $15 \mathrm{q} 13.3$ deletion. There was no sex bias for ascertainment outside of that observed for transmitting parents, suggesting there may be differential negative effects of the 15q13.3 deletion on the reproductive fitness of men as reported for $22 \mathrm{q} 11.2$ deletions. ${ }^{28}$

A further novel finding was the implication of placenta previa as a potential feature of $15 q 13.3$ deletions, with a prevalence $(1.6 \%)$ in probands up to fivefold greater than standard population estimates (2.9 per 1,000 births). ${ }^{29}$ The paucity of obstetric history available for individuals with 15q13.3 deletions, however, meant that detailed investigation of a possible connection, including confounding risk factors such as advanced maternal age and smoking during pregnancy, ${ }^{30}$ was beyond the scope of this study.

\section{Potential genotype-phenotype correlations}

Nested 15q13.3 deletions overlapping only the CHRNA7 gene, encoding a ligand-gated cation channel mediating neuronal transmission, have been found in individuals with DD/ID, ASD, epilepsy, and schizophrenia, thus implicating CHRNA7 as a top candidate gene for the neuropsychiatric manifestations of the 15q13.3-deletion syndrome. ${ }^{18,31,32}$ However, defining a "critical region" or critical genes for 15q13.3 microdeletion expression remains challenging. A recent study showed significant enrichment of multiple rare, nonsynonymous variants spanning a 20-kb region overlapping FAN1 in schizophrenia and ASD as compared with controls, thus implicating this gene, which encodes a DNA repair nuclease, as another potential contributor to the 15 q13.3-deletion neuropsychiatric phenotype. ${ }^{33}$
Other BP4-BP5 region genes with brain expression include TRPM1, encoding a calcium permeable cation channel. TRPM1 is a leading candidate for the visual impairment in children with homozygous 15q13.3 deletions, given its involvement in heterogeneous autosomal recessive forms of complete congenital stationary night blindness (CSNB1C, OMIM 613216).$^{34}$ Notably, we found no reports of comparable visual impairment for any individual with heterozygous 15q13.3 deletions, although one child did have microphthalmia. Three additional cases with 15q13.3 homozygous deletions reported in 2014 provide some support for the likelihood that biallelic loss of TRPM1 may be associated with characteristic retinal dysfunction. ${ }^{20,27}$ However, cases with deletions showing biallelic loss of only CHRNA7 also have visual impairment, thus limiting definitive conclusions..$^{20,27}$

With respect to placenta previa, there are studies of embryo implantation in a null mouse model implicating the $15 q 13.3$ region gene $K L F 13$, as well as $K L F 9$, a related gene located at 9q13. ${ }^{35}$ These genes encode members of the Krüppel-like family of transcription factors, which are needed to maintain the proper progesterone sensitivity regulation that is essential for successful embryo implantation. ${ }^{36}$ Further study is required to understand the role of KLF13 in human perinatal outcomes. This may include sequencing the intact copy of KLF13 in 15q13.3-deletion cases with and without adverse perinatal outcomes and/or infertility or early loss (Table 1; Figure 2).

\section{Clinical implications}

The results of this study may help guide genetic counseling and anticipatory care for individuals with 15q13.3 deletions. The available evidence suggests that the majority of individuals with a 15q13.3 deletion will develop one or more neurodevelopmental and/or neuropsychiatric conditions. Developmental assessments and neurocognitive testing would be essential to determining the educational assistance and other services, such as early childhood programs, that could help to maximize developmental outcomes. Electroencephalography and a neurological examination are also recommended at time of diagnosis and periodically at follow-up. Speech assessment and therapy would benefit patients manifesting speech difficulties. Genetic counseling should also highlight the high prevalence of neuropsychiatric conditions so that the patient, family members, primary physician, and other caregivers can be vigilant in watching for changes in mood, thinking, and/or behavior that may herald the onset of a treatable psychiatric disorder.

Although rare, the 15q13.3-deletion syndrome may include congenital cardiac defects; therefore, echocardiography should be considered where indicated. Growth abnormalities (both undergrowth and overgrowth) may occur. Additional confirmatory data and comparison to population norms are needed to determine whether endocrine/metabolic conditions may be associated with the 15q13.3 deletion (Table 1). Individuals with the $15 \mathrm{q} 13.3$ deletion should be counseled on the $50 \%$ risk of transmitting the deletion. In addition to standard genetic counseling, women (affected and unaffected) carrying a fetus with the $15 q 13.3$ deletion should be monitored and considered for 
referral to a specialist if complications such as placenta previa are identified. Because of the likelihood that many (if not most) 15q13.3 deletions are inherited, parental studies using array comparative genomic hybridization, multiplex ligation-dependent probe amplification, or fluorescence in situ hybridization (using a 15q13.3 region probe such as BAC RP11-717124), as well as testing of other relatives where relevant, should be carried out.

The as yet limited data available for the BP3-BP4 deletion, especially for adults, preclude conclusions about its penetrance and expressivity. However, preliminary results based mainly on the ascertainment phenotype suggest that genetic counseling for this rare, recurrent $\mathrm{CNV}$ continue to highlight the risk for DD/ID and other neuropsychiatric features.

\section{Advantages and limitations}

Advantages of this study include our systematic approach, for example, examining supplementary material and using multiple cases across several ascertainment disorders and relatives to delineate the expressivity and penetrance of the 15q13.3 deletion. ${ }^{8}$ We did not rely solely on ascertainment data to determine the prevalence of various disorders associated with the 15q13.3 deletion but rather categorized individual features for each case in the sample so that the overall prevalence for each feature could be determined.

Limitations included the challenge of obtaining detailed clinical information for subjects, leading to potential underestimation of certain conditions and features (e.g., prenatal, lateronset, dysmorphic). In addition, because the majority of our cases came from the literature, it is likely that some conditions were overestimated (e.g., conditions used for ascertainment) and others were not reported at all, as was sometimes the case for even basic demographic variables (e.g., age, sex, ethnicity). Furthermore, few subjects had formal cognitive testing, limiting our ability to draw specific conclusions about the neurocognitive profile of cases with the 15q13.3 deletion.

\section{Future directions}

As for other microdeletion syndromes, the challenge will be to develop an explanation for the observed phenotypic variability and incomplete penetrance of the $15 \mathrm{q} 13.3$ microdeletion. ${ }^{37}$ Several genetic and nongenetic hypotheses have been proposed to explain this variability, including (i) sequence variants on the intact chromosome, (ii) sequence variants elsewhere in the genome, (iii) genetic background, (iv) position effects, (v) epigenetic processes, and (vi) environmental influences.$^{37}$ In addition, regulatory elements (e.g., microRNAs) may play a role in mediating the risk for neurodevelopmental disorders. ${ }^{38,39}$ Interestingly, two microRNAs, miR-211 and miR-4509, are overlapped by the typical BP4-BP5 and larger BP3-BP4 15q13.3 deletions, respectively (Figure 1 ). Further study that likely involves available registries and consortia capable of providing large samples, includes detailed phenotyping of children and adults, and investigates issues such as reproductive fitness will be important for improved understanding of 15q13.3 deletions.

\section{Conclusions}

15q13.3 Deletions involving BP4-BP5 appear to present a significant risk for the expression of neuropsychiatric conditions, including DD/ID, ASD, epilepsy, schizophrenia, ADHD, and behavioral problems. The collective penetrance of one or more of these features may approach $80 \%$. The majority of these deletions are inherited, most often maternally, and yet are rare in control populations. Reduced reproductive fitness in males may explain the excess of maternally inherited 15q13.3 deletions. A newly identified potential feature of the 15q13.3-deletion syndrome is placenta previa. Further studies, including detailed cross-sectional assessment of patients of all ages and longitudinal follow-up, are needed to inform genetic counseling and anticipatory care.

\section{SUPPLEMENTARY MATERIAL}

Supplementary material is linked to the online version of the paper at http://www.nature.com/gim

\section{ACKNOWLEDGMENTS}

The authors thank the patients and their families for their participation; research assistants and staff at Saint John Community Mental Health Services and the Saint John Regional Hospital; S. Bekeschus for figure preparation; and N. Butcher and D.A. Baribeau for revising the manuscript. Study support was provided by Canadian Institutes of Health Research grants (MOP 111238 and MOP 89066 to A.S.B.). A.S.B. holds the Canadian Research Chair in Schizophrenia Genetics and Genomic Disorders and the Dalglish Chair in 22q11.2 Deletion Syndrome. Acknowledgments for control data sets are in Supplementary Table S7 online.

\section{DISCLOSURE}

S.W.S. is on the Scientific Advisory Board of Population Diagnostics and is a cofounder of YouNique Genomics. The other authors declare no conflict of interest.

\section{REFERENCES}

1. Pujana MA, Nadal M, Guitart M, Armengol L, Gratacòs M, Estivill X. Human chromosome 15q11-q14 regions of rearrangements contain clusters of LCR15 duplicons. Eur J Hum Genet 2002;10:26-35.

2. Bassett AS. Parental origin, DNA structure, and the schizophrenia spectrum. Am J Psychiatry 2011;168:350-353.

3. Sharp AJ, Mefford HC, Li K, et al. A recurrent 15q13.3 microdeletion syndrome associated with mental retardation and seizures. Nat Genet 2008;40:322-328.

4. van Bon BW, Mefford HC, Menten B, et al. Further delineation of the $15 q 13$ microdeletion and duplication syndromes: a clinical spectrum varying from nonpathogenic to a severe outcome. J Med Genet 2009;46:511-523.

5. Helbig I, Mefford HC, Sharp AJ, et al. 15q13.3 microdeletions increase risk of idiopathic generalized epilepsy. Nat Genet 2009;41:160-162.

6. Costain $\mathrm{G}$, Lionel $A C$, Merico $D$, et al. Pathogenic rare copy number variants in community-based schizophrenia suggest a potential role for clinical microarrays. Hum Mol Genet 2013;22:4485-4501.

7. Miller DT, Shen Y, Weiss LA, et al. Microdeletion/duplication at 15q13.2q13.3 among individuals with features of autism and other neuropsychiatric disorders. J Med Genet 2009;46:242-248.

8. Dolcetti A, Silversides CK, Marshall CR, et al. 1q21.1 Microduplication expression in adults. Genet Med 2013;15:282-289.

9. Kaminsky EB, Kaul V, Paschall J, et al. An evidence-based approach to establish the functional and clinical significance of copy number variants in intellectual and developmental disabilities. Genet Med 2011;13:777-784. 
10. Miller DT, Adam MP, Aradhya S, et al. Consensus statement: chromosomal microarray is a first-tier clinical diagnostic test for individuals with developmental disabilities or congenital anomalies. Am J Hum Genet 2010;86:749-764.

11. Scott F, Murphy K, Carey L, et al. Prenatal diagnosis using combined quantitative fluorescent polymerase chain reaction and array comparative genomic hybridization analysis as a first-line test: results from over 1000 consecutive cases. Ultrasound Obstet Gynecol 2013;41:500-507.

12. Silversides CK, Lionel AC, Costain $G$, et al. Rare copy number variations in adults with tetralogy of Fallot implicate novel risk gene pathways. PLoS Genet 2012;8:e1002843.

13. Fung WL, Chow EW, Webb GD, Gatzoulis MA, Bassett AS. Extracardiac features predicting 22q11.2 deletion syndrome in adult congenital heart disease. Int J Cardio/ 2008; 131:51-58.

14. Bushby KM, Cole T, Matthews JN, Goodship JA. Centiles for adult head circumference. Arch Dis Child 1992;67:1286-1287.

15. Health Canada. Canadian Perinatal Health Report, 2008. Minister of Public Works and Government Services Canada: Ottawa, Canada, 2008.

16. Kramer MS, Platt RW, Wen SW, et al.; Fetal/Infant Health Study Group of the Canadian Perinatal Surveillance System. A new and improved populationbased Canadian reference for birth weight for gestational age. Pediatrics 2001;108:E35.

17. Firth HV, Richards SM, Bevan AP, et al. DECIPHER: Database of Chromosomal Imbalance and Phenotype in Humans Using Ensembl Resources. Am J Hum Genet 2009;84:524-533.

18. Masurel-Paulet A, Andrieux J, Callier P, et al. Delineation of 15q13.3 microdeletions. Clin Genet 2010;78:149-161.

19. Spielmann M, Reichelt G, Hertzberg, et al. Homozygous deletion of chromosome 15q13.3 including CHRNA7 causes severe mental retardation, seizures, muscular hypotonia, and the loss of KLF13 and TRPM1 potentially cause macrocytosis and congenital retinal dysfunction in siblings Eur J Med Genet 2011;54:e441-e445.

20. Masurel-Paulet A, Drumare I, Holder M, et al. Further delineation of eye manifestations in homozygous 15q13.3 microdeletions including TRPM1: a differential diagnosis of ceroid lipofuscinosis. Am J Med Genet A 2014;164A:1537-1544

21. Stefansson $H$, Meyer-Lindenberg A, Steinberg S, et al. CNVs conferring risk of autism or schizophrenia affect cognition in controls. Nature 2014;505:361366.

22. Mefford HC, Muhle H, Ostertag P, et al. Genome-wide copy number variation in epilepsy: novel susceptibility loci in idiopathic generalized and focal epilepsies. PLoS Genet 2010;6:e1000962.

23. Wapner RJ, Martin CL, Levy B, et al. Chromosomal microarray versus karyotyping for prenatal diagnosis. N Engl J Med 2012;367:2175-2184.

24. Fiorentino F, Napoletano S, Caiazzo F, et al. Chromosomal microarray analysis as a first-line test in pregnancies with a priori low risk for the detection of submicroscopic chromosomal abnormalities. Eur J Hum Genet 2013;21:725-730.

25. Bassett AS, McDonald-McGinn DM, Devriendt K, et al. Practical guidelines for managing patients with 22q11.2 deletion syndrome. J Pediatr 2011;159:332339 e331.
26. Costain G, McDonald-McGinn DM, Bassett AS. Prenatal genetic testing with chromosomal microarray analysis identifies major risk variants for schizophrenia and other later-onset disorders. Am J Psychiatry 2013;170:1498.

27. Prasun P, Hankerd M, Kristofice M, Scussel L, Sivaswamy L, Ebrahim S. Compound heterozygous microdeletion of chromosome $15 q 13.3$ region in a child with hypotonia, impaired vision, and global developmental delay. Am J Med Genet A 2014;164:1815-1820.

28. Costain G, Chow EW, Silversides CK, Bassett AS. Sex differences in reproductive fitness contribute to preferential maternal transmission of $22 q 11.2$ deletions. $J$ Med Genet 2011;48:819-824.

29. Cresswell JA, Ronsmans C, Calvert C, Filippi V. Prevalence of placenta praevia by world region: a systematic review and meta-analysis. Trop Med Int Health 2013;18:712-724.

30. Rao KP, Belogolovkin V, Yankowitz J, Spinnato JA 2nd. Abnormal placentation: evidence-based diagnosis and management of placenta previa, placenta accreta, and vasa previa. Obstet Gynecol Surv 2012;67:503-519.

31. Hoppman-Chaney N, Wain K, Seger PR, Superneau DW, Hodge JC Identification of single gene deletions at 15q13.3: further evidence that CHRNA7 causes the 15q13.3 microdeletion syndrome phenotype. Clin Genet 2013;83:345-351.

32. Liao J, DeWard SJ, Madan-Khetarpal S, Surti U, Hu J. A small homozygous microdeletion of 15q13.3 including the CHRNA7 gene in a girl with a spectrum of severe neurodevelopmental features. Am J Med Genet A 2011;155A:27952800

33. Ionita-Laza I, Xu B, Makarov V, et al. Scan statistic-based analysis of exome sequencing data identifies FAN1 at $15 q 13.3$ as a susceptibility gene for schizophrenia and autism. Proc Natl Acad Sci USA 2014;111: 343-348

34. Audo I, Kohl S, Leroy BP, et al. TRPM1 is mutated in patients with autosomalrecessive complete congenital stationary night blindness. Am J Hum Genet 2009;85:720-729.

35. Heard ME, Pabona JM, Clayberger C, Krensky AM, Simmen FA, Simmen RC. The reproductive phenotype of mice null for transcription factor Krüppel-like factor 13 suggests compensatory function of family member Krüppel-like factor 9 in the peri-implantation uterus. Biol Reprod 2012;87:115.

36. Pabona JM, Zeng Z, Simmen FA, Simmen RC. Functional differentiation of uterine stromal cells involves cross-regulation between bone morphogenetic protein 2 and Kruppel-like factor (KLF) family members KLF9 and KLF13. Endocrinology 2010;151:3396-3406.

37. Costain G, Bassett AS. Clinical applications of schizophrenia genetics: genetic diagnosis, risk, and counseling in the molecular era. App/ Clin Genet 2012;5: $1-18$.

38. Brzustowicz LM, Bassett AS. miRNA-mediated risk for schizophrenia in 22q11.2 deletion syndrome. Front Genet 2012;3:1-9.

39. Mellios N, Sur M. The emerging role of microRNAs in schizophrenia and autism spectrum disorders. Front Psychiatry 2012;3:1-9.

40. MacDonald JR, Ziman R, Yuen RK, Feuk L, Scherer SW. The Database of Genomic Variants: a curated collection of structural variation in the human genome. Nucleic Acids Res 2014:42(Database issue):D986-D992. 